\title{
LEPROMATOUS NEURITIC LESIONS, THEIR GRIEAT INCIDENCE IN CERTAIN SENSORY $\Lambda$ NI) MO'TOR BRANCHES, AND THE TREATMENT
}

Dr. Mario Guadagnini.

\section{LePromatous Neuritic Lesions}

We wish to refer to the hypertrophic lepromatous neuritic lesions and their pain symptomatology, which is a clinical aspect bound up with anatomical factors. We propose to describe the surgical form of attack on the neural and paraneural picture.

The cases which we present were taken in the acute or subacute period, when the nerve feels enlarged, painful, tense, and of increased consistency, with the surface apparently regular and even, or deformed by the presence of circumscribed nodules which occupy the inner nerve. The tendency which certain sensory-motor nerve branches have to localize the processes of reactional hypertrophic neuritis in particular parts of their course, we interpret as being related to the existence of anatomical paraneural factors which affect the nerve in areas contiguous to the articulations: there the muscle masses disappear, being substituted by ligamentous insertions; there the proximity of osseous planes and aponeuroses do not permit of a uniform distension of the nerve in the first stages of the diffuse neuritis, so that the motor branches, in spite of undergoing the same blood invasion during the reactional bacillaemias, do not give any appreciable painful symptomatology. This condition one supposes is due to their not having suffered compression in their course.

The sensory-motor nerves which become tender and hypertrophic are, in order of frequency, the cubital, which suffers compression in its passage through the inner region of the elbow as it enters the epitrochlear-olecranon tunnel: then the external popliteal when it turns over the head of the fibula where it is imprisoned by the aponeurosis; then the median nerve which is exposed to the same conditions in the zone of wrist flexion as it crosses the anterior annular ligament of the wrist: finally the posterior tibial nerve in its passage through the tunnel formed by the internal tarsal ligament in the internal retro--malleolar region. It is always above the compressed portions of the nerve where occurs the enlargement of the nerve, with a dilatation of the epineural capillaries inducing a varicose aspect, accompanied by a stagnation of blood circulation in the neural vessels which adds to the active local congestion. This 
situation contrasts on the other hand with that of the compressed part of the nerve, which remains free of congestion and thickening. For this reason we do not limit our intervention to freeing the nerve, but seek to eliminate or relieve also the paraneural factors which cause the compression.

\section{Surgical Considerations}

Surgical intervention comprises the isolation of the nerve, its decapsulation and neurolysis, with elimination of the factors causing neural compression.

The nerve is approached by an incision which embraces the thickened tender part together with the compressed sector. Having incised the superficial planes and isolated the nerve, two sutures are passed below the nerve and brought to the extremities with the object of holding the nerve firm in the procedure of neurolysis.

The paraneural aponeurotic tissues are incised in the whole area of compression of the nerve so as to attain its liberation, so that for the cubital one opens the epitrochlear tissue and its aponeurotic prolongation of the anterior cubital muscle; for the external popliteal one opens the tibio-peroneal aponeurosis; for the median nerve. the anterior annular carpal ligament; for the posterior tibial, the internal tarsal ligament.

Having decompressed the nerve, one begins the neurolysis with the decapsulation by means of a longitudinal incision of the epineurium or sheath, including the endoneurium which is divided almost in two halves, all this being carried out with the nerve held tense by means of the two sutures placed at the ends. The epineurium is dissected and separated from the underlying plane of the endoneurium, on which are carried out other longitudinal incisions which are adjusted to the degree of thickening of the nerve. Having completed the neurolysis, we are accustomed to reconstruct the epineurium in the whole of its decapsulated course with interrupted stitches. Finally, as suggested by Dr. Robert G. Cochrane during his visit to the Sanatorium Sommer, in two cases we have carried out total resection of the epineurium, with good results.

We think it of interest to detail some particulars of technique relating to the various sensory-motor branches concerned, beginning with the cubital nerve, which provides the highest percentage of hypertrophic neuritis. In order to attack this nerve we place the patient on the operating table in the supine position, with his arm extended and rotated forward so as to expose the epitrochlearolecranon region. The incision extends from the middle third or lower third of the inner surface of the arm towards the hypertrophied portion of the nerve, and is carried on as far as the forearm in a 
curved line above the course of the epitrochlear-olecranon furrow. The skin having been incised and the superficial fascia exposed, one makes an opening immediately above the epitrochlear tendon or entrance to the tunnel in order to expose the nerve to view, and where is introduced the half-opened scissors in order to push it upwards and open up the internal intramuscular partition wall of the arm, a space which the cubital nerve traverses. With a similar manœuvre in the opposite way towards the forearm one opens the epitrochlear corridor in its whole length as far as the fibres of the anterior cubital muscle. . With this technique one simplifies and facilitates the isolation of the nerve in all its affected course, including the compressed portion, without damaging it by laborious dissections, especially when the reactionary perineural processes generate organized adhesions to the surrounding tissue planes, as happens in the case of the cubital nerve in the middle or lower third of the arm.

Having opened the epitrochlear-olecranon tunnel (the cause of the nerve compression because of its anatomical characteristics of being in the form of an inextensible osteo-fibrous tube and having a scanty lining of adipose tissue), the nerve is freed and loosened from the narrow bed formed by the tunnel: then the nerve is displaced and transposed in front of the internal condyle or epitrochlea. For that purpose, previously, the cutaneous plane which covers the internal surface of the elbow in relation to the epitrochlea is freed from the underlying superficial muscular fascia, so as to make a place for the new bed which the cubital nerve should now have in exchange for its pre-epitrochlear course.

The transposed cubital nerve is fixed in front of the epitrochlea, making use of the cellular fascia, which we dissect from the internal attachments of the elbow. In its new course the nerve shortens its curve, its stretchings in the movements of arm flexion are diminished, and the compressions due to the tunnel are eliminated.

Concerning the external popliteal nerve, which is frequently involved, it can be felt thickened within the biceps-crural muscle in the region of the popliteal fossa. Here the phenomena of compression occur when the nerve turns through half a circle about the head of the fibula, a place where the tibio-peroneal aponeurosis flattens it against the bony surface, so that the nerve takes a tapelike form. In the operation, the tibial aponeurosis is incised up to the bifurcation of the nerve into its two branches, the anterior tibial and the musculo-cutaneous, among the fibres of the long lateral peroneal muscle, respecting as far as possible the branch of the cutaneous peroneal which starts further up. At the end of the operation the neurolysis is completed with the partial resection of the 
edges of the tibial aponeurosis, which are left without being sutured.

The median nerve localizes the processes of neuritis in the flexor aspect of the wrist, and the circumscribed and fusiform hypertrophy of the nerve can be felt among the major and minor palmar tendons and sometimes be made to stand out when the wrist is placed in hyperextension. The compression phenomena are produced when the nerve passes through the medio-carpal canal, along with the flexor tendons of the hand; this canal is formed by the anterior annular ligament of the wrist, which surrounds the nerve and the tendons in a fibrous semi-circle. In the operation the said ligament, which is tough and firm, is incised in its whole breadth and the incision goes from the lower part of the wrist to the base of the palm, where again the nerve has a degree of freedom.

Finally the posterior tibial nerve, sometimes forgotten.in clinical examination, is felt tender and increased in volume between the internal malleolus and the tendo Achilles; in its course through the tissues of the instep it is accompanied by the tibial vessels until it reaches the internal tarsal ligament. This ligament is formed by the fusion of the superficial and deep aponeurosis of the region, which establish four fibrous tunnels, in one of which the nerve is imprisoned by the deep planes formed by the calcaneus bone, and it is in this course that it is first subject to compression in the neuritic reactions. In the freeing of the nerve the tarsal ligament is opened in the whole course of the tunnel, in its characteristic curved course.

\section{Conclusion and Statistics}

In the Sanatorium Sommer de Rodriguez, Argentina, there have been carried out ig'8 surgical operations for neuritis, based on the different clinical forms and evolutionary stages of all the cases subjected to operation, I46 correspond to the acute or subacute reactional lepromatous type with painful hypertrophy, of variable intensity, and preceded by cutaneous activity in the form of erythema nodosum or polymorphic erythema, the clinical type with which we have been concerned in this paper. The distribution of the operations for neurolysis and decompression on the various sensory-motor nerves is as follows:-

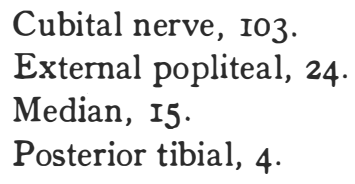

The patients subjected to operation had been suffering from neuritis of painful nature from a few days to a year or more. Some had had partial remissions followed by sharp new exacerbations, of which opportunity was taken to intervene. 
The pain-removing effect of the decompression and neurolysis was immediate in all the cases above the hypertrophied part of the nerve. The distal disturbances, such as areas of painful hyperaesthesia, parasthaesia, and cutaneous blunting of sensation, showed less apparent favourable modifications, but were appreciable mostly in the post-operative period, with diminution or clisappearance of the pains in 50 to $5.5 \%$.

In this account we have not made note of trophic modifications conseguent on freeing the nerve.

In the total of those operated on we have had 24 relanses of pain, in patients between the first and third yoar and in whom it was necessary to reintervene, also patients with marked cutaneous artivity. The natire of the relapse's was that there symptoms were milerer with less pain and the inflammatory reaction was more circumscribed in the affected area.

The listribution of the relapses was:-

$$
\begin{aligned}
& \text { Cubital, 17. } \\
& \text { Iixternal popliteal, } 5 . \\
& \text { Median, } 2 . \\
& \text { Posterior tibial, o. }
\end{aligned}
$$

A second operation re-established calm, excent for 2 cases of neuritis of the cubital which required a third operation.

Summary. There are certain sensory-motor nerve's which are greatly predisposed to the processes of painful hypertrophic neuritis.

We interpret the pathology as bound up with certain anatomical perineural relations constituted by aponeurosis, ligaments, and osseous planes which act as compression factors on the nerve in the initial processes of the diffuse neuritis.

These painful forms of neuritis are suitable for surgical intervention, which is a treatment directed to the relief of pain and the amelioration of the trophic lesions. 'To this end are carried ont decapsulation and neurolysis, with elimination of the perineural anatomical factors in compression.

The pain-removing action in the operated portion of the nerve is immediate in all patients: this is less marked in distal disturbances. With regard to the distal manifestations corresponding to the nerve branch affected, the improvement varies with the timeliness of the operation and the valuable effects of post-operative care.

Notr. - The photographs have been made by the Rev. Chaplain of the Sanatorium Sommer, Señor Joaquín Prochaka, to whom my thanks are due.

(Translation from the oxrigiral Spanish. --J.R.I.) 


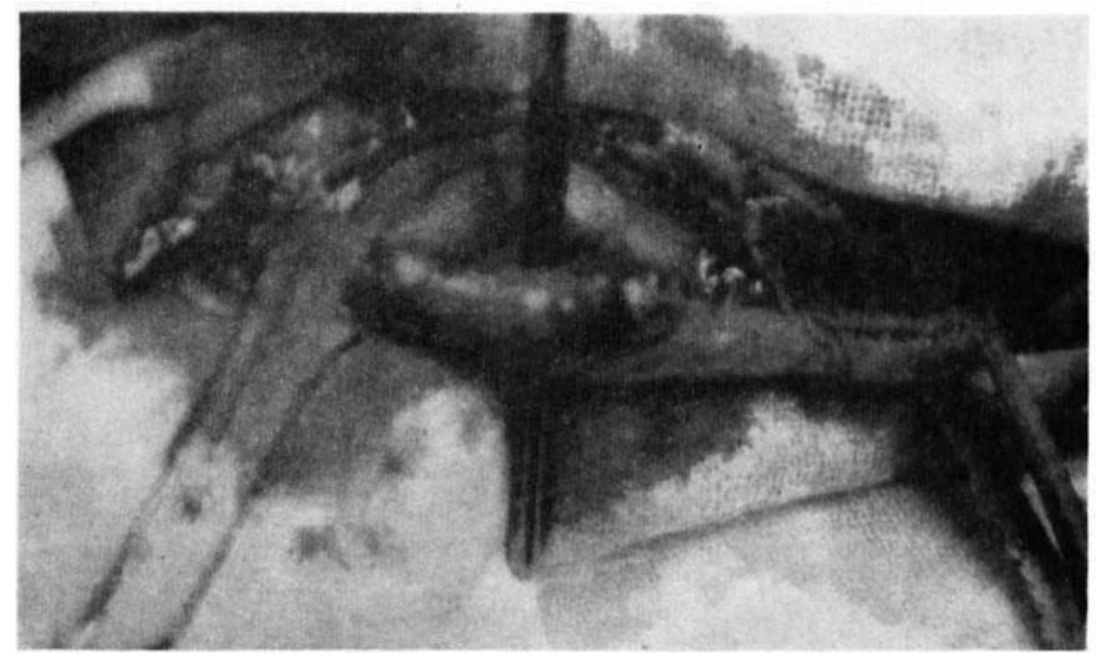

FigitiR: N.1-Ulnat nerve in reaction, with two nodules projecting and multiple vessels with a varicose appearance.

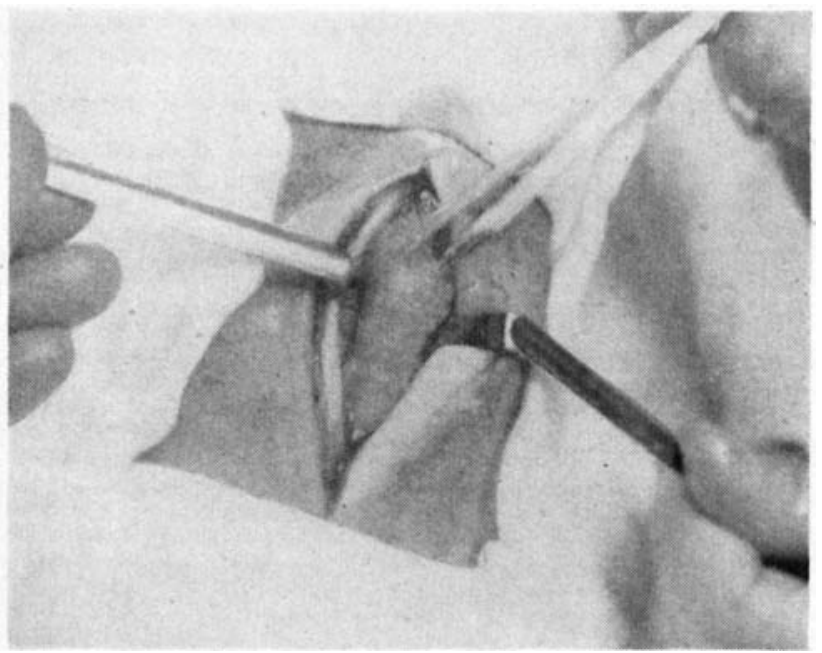

Figurf: N.2- Hypertrophied median nerve above the annular ligament on the anterior aspect. 


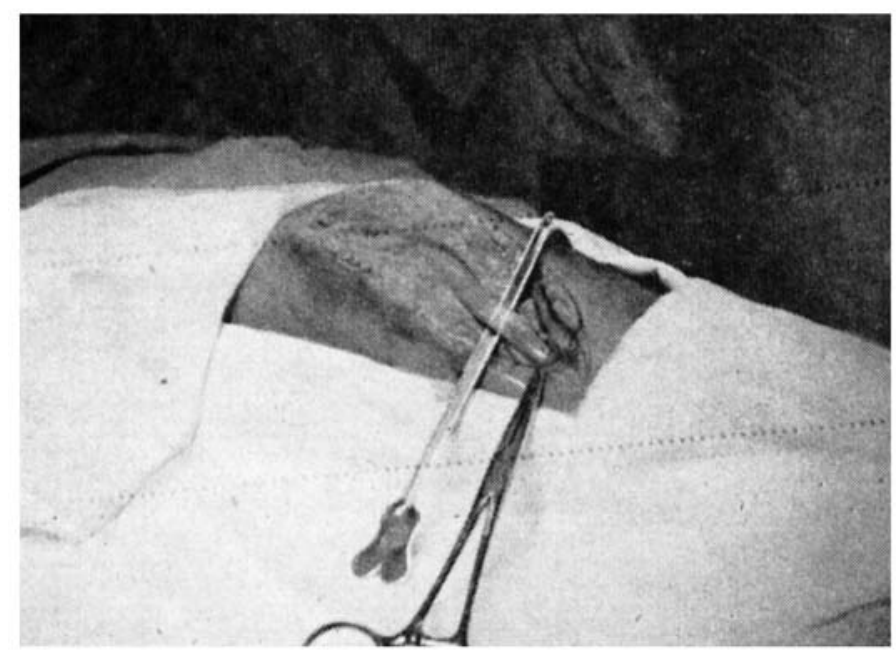

Figure N.3-Hypertrophied ulnar nerve on the epitrochlear condyle.

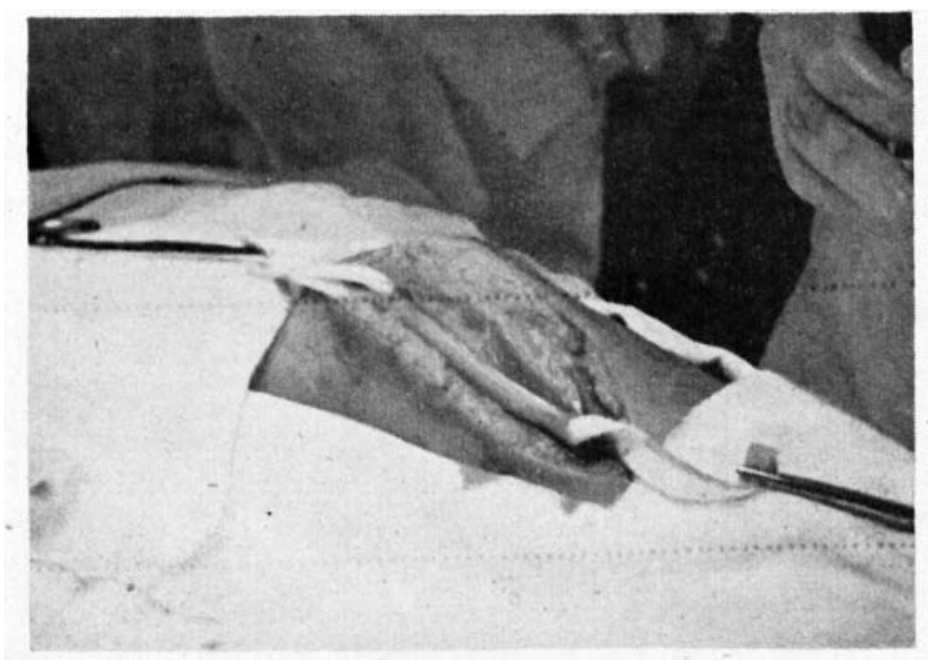

Figure N.4-Hypertrophied ulnar nerve displayed from its bed. 


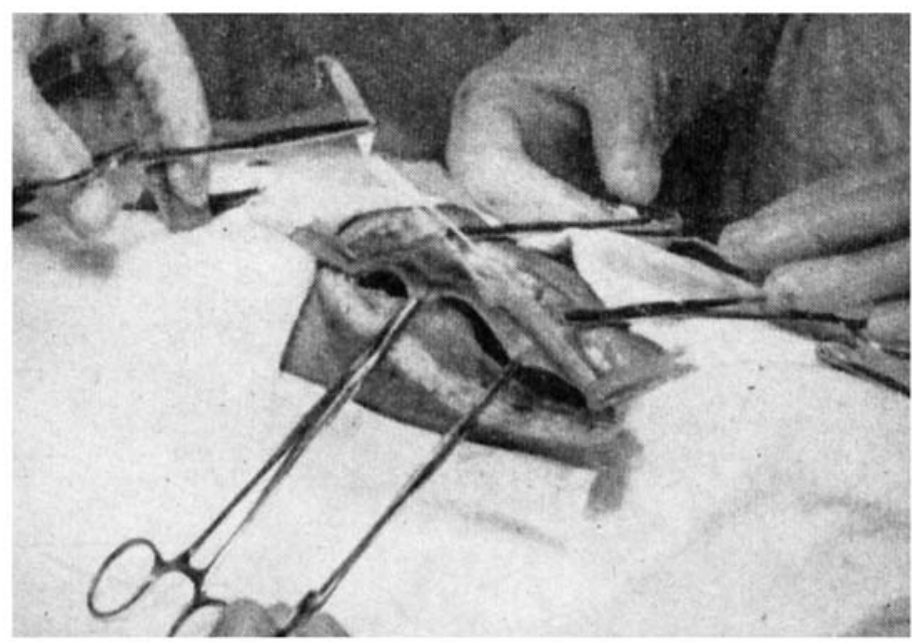

Figur: N.5-Ulnar nerve with the epincurium opened.

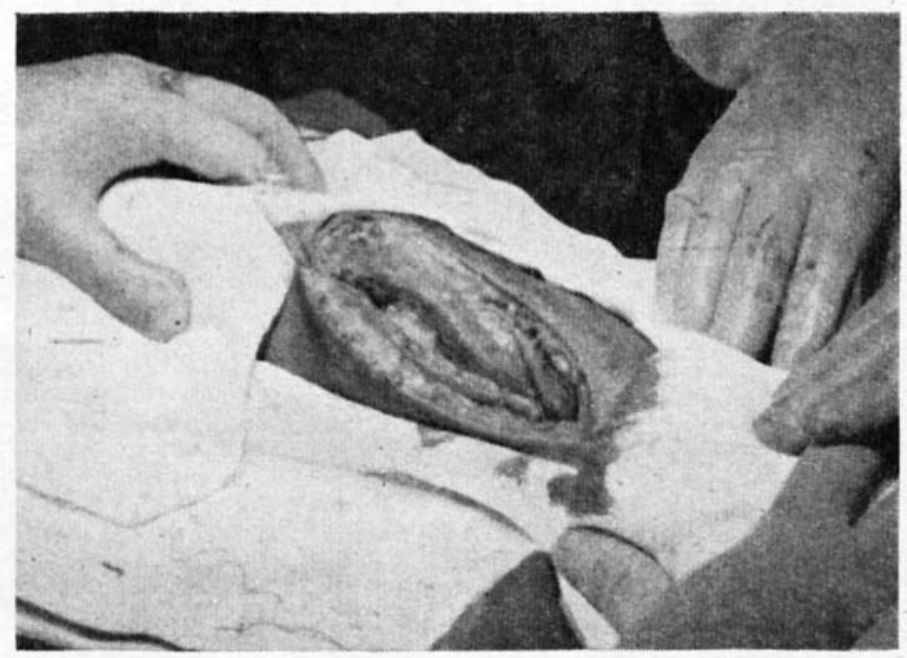

. Figure N.G-Ulnar nerve with its epineurium reconstructed. 


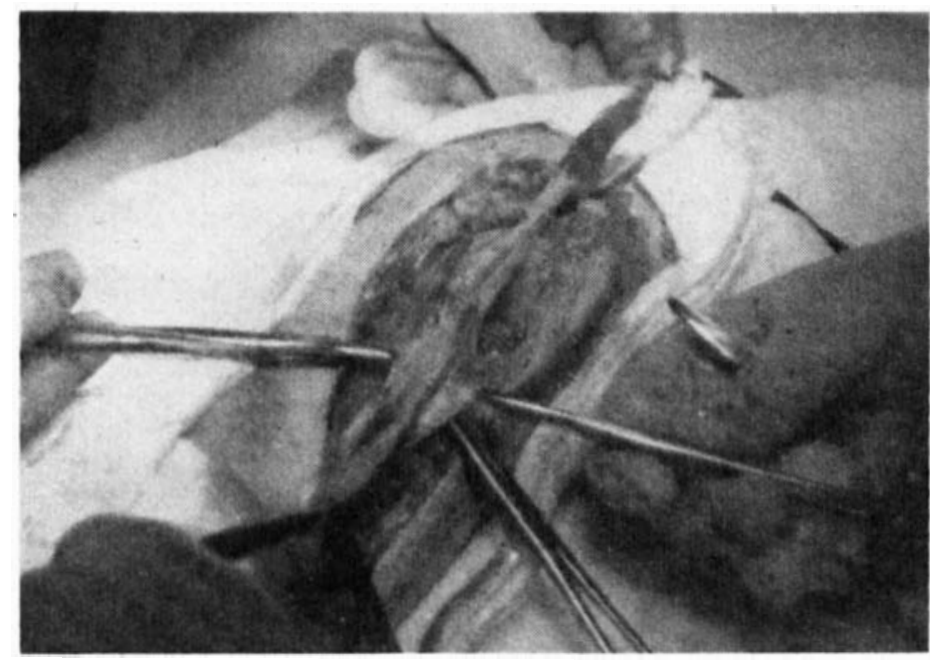

Figuri: N.8-lixternal popliteal nerve with the capsule removed. Notice the flattening of the part which passes round the head of the tibul:1.

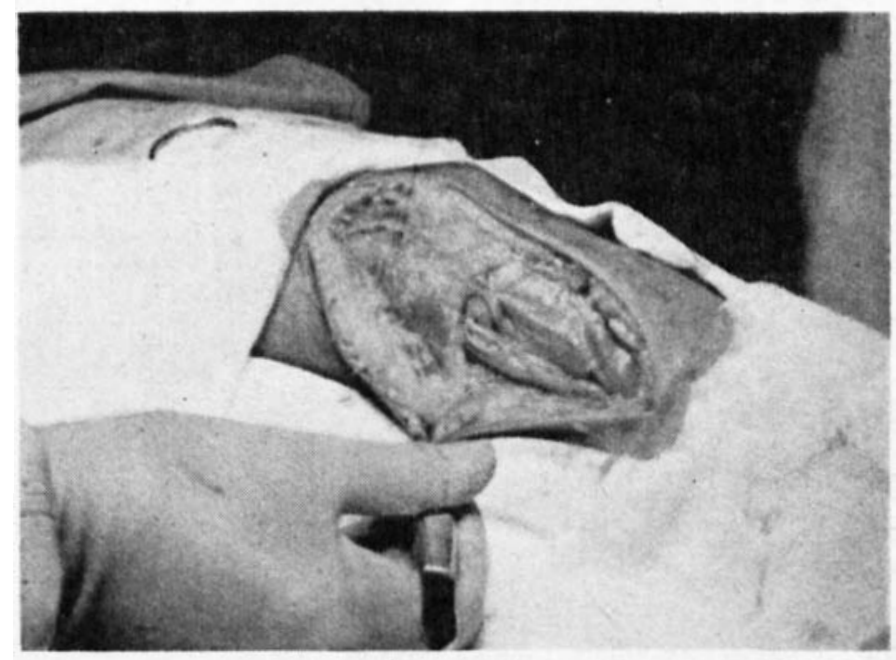

Figurf N.7-Ulnar nerve displaced, and fixed by a portion of cellular fascia. 